\title{
A LOCAL RESULT FOR SYSTEMS OF RIEMANN-HILBERT BARRIER PROBLEMS $\left({ }^{1}\right)$
}

BY

KEVIN F. CLANCEY

ABSTRACT. The Riemann-Hilbert barrier problem (for $n$ pairs of functions)

$$
G \Phi^{+}=\Phi^{-}+g
$$

is investigated for the square integrable functions on a union of analytic Jordan curves $C$ bounding a domain in the complex plane. In the special case, where at each point $t_{0}$ of $C$ the symbol $G$ has at most two essential cluster values $G_{1}\left(t_{0}\right), G_{2}\left(t_{0}\right)$, then the condition $\operatorname{det}\left[(1-\lambda) G_{1}\left(t_{0}\right)+\lambda G_{2}\left(t_{0}\right)\right] \neq 0$, for all $t_{0}$ in $C$ and all $\lambda(0<$ $\lambda<1$ ), implies the Riemann-Hilbert operator is Fredholm. In the case, where for some $t_{0}$ in $C$ and some $\lambda_{0}\left(0<\lambda_{0}<1\right)$, $\operatorname{det}\left[\left(1-\lambda_{0}\right) G_{1}\left(t_{0}\right)+\lambda_{0} G_{2}\left(t_{0}\right)\right]=0$, the Riemann-Hilbert operator is not Fredholm. An application is given to systems of singular integral equation on $L^{2}(E)$, where $E$ is a measurable subset of $C$.

Let $D^{+}$be an open connected region in the complex plane $\mathbf{C}$ with boundary $C$ consisting of $m+1$ nonintersecting rectifiable analytic Jordan curves $C_{0}, C_{1}, \cdots, C_{m}$. The domain complementary to $D^{+} \cup C$ is denoted by $D^{-}$. It is assumed that $C_{0}$ is the boundary of the unbounded component of $D^{-}$ and that the boundary $C$ is oriented positively with respect to $D^{+}$. The notation $L_{n}^{p}(C)(1 \leqslant p<\infty)$ will be used for the Lebesgue spaces of $\mathbf{C}^{n}$. valued, $p$-integrable (with respect to arc length measure on $C$ ) functions on $C$. In the case $p=\infty, L_{n}^{\infty}(C)$ will denote the essentially bounded $\mathbf{C}^{n}$-valued measurable functions on $C$. If $A$ is any nonempty set, then $A_{M_{n}}$ will stand for the collection of $n \times n$ matrices with entries from $A$.

For $f$ in $L_{n}^{p}(C)(p \geqslant 1)$ we define the Cauchy transform of $f$ by $C f(z)=(2 \pi i)^{-1} \int_{C} f(t)(t-z)^{-1} d t, \quad z \notin C$. Obviously, $C f$ is a $\mathbf{C}^{n}$-valued function separately analytic in $D^{+}$and $D^{-}$. Moreover, the nontangential limits of $C f$ from $D^{+}$and $D^{-}$exist a.e. on $C$. These nontangential limits $f^{ \pm}$satisfy a.e. the Plemelj identities

$$
\begin{aligned}
& f^{+}(t)-f^{-}(t)=f(t), \\
& f^{+}(t)+f^{-}(t)=(\pi i)^{-1} \int_{C} f(s)(s-t)^{-1} d s .
\end{aligned}
$$

Received by the editors October 24, 1973.

AMS (MOS) subject classifications (1970). Primary 43E05; Secondary 47E05.

(1) This work was supported by the National Science Foundation Grant \#GP 38488.

Copyright @ 1974, American Mathematical Society 
The singular integral $Q f(t)=(\pi i)^{-1} \int_{C} f(s)(s-t)^{-1} d s$ is interpreted as a Cauchy principal value. For the definition of the Cauchy principal value the reader is referred to Muskhelishvili $[10$, p. 25]. Further, it is an easy consequence of M. Riesz's theorem on the boundedness of the conjugate integral (see e.g. Zygmund $\left[14\right.$, p. 253]) that the map $f \rightarrow Q f$ is bounded on $L_{n}^{p}(C)$, for any $p>1$. Of course, this implies that the maps $f \rightarrow f^{ \pm}$are bounded on $L_{n}^{p}(C)$; for any $p>1$.

Let $G$ be in $L_{M_{n}}^{\infty}(C)$. We are interested in the Riemann-Hilbert operator $R_{G}: L_{n}^{p}(C) \rightarrow L_{n}^{p}(C)(p>1)$ defined by

$$
R_{G} f=G f^{+}-f^{-} \text {. }
$$

If $g$ is in $L_{n}^{p}(C)$, then the problem of solving the equation $R_{G} f=g$ in $L_{n}^{p}(C)$ is equivalent to finding a $\mathbf{C}^{n}$-valued function $\Phi$ separately analytic in $D^{+}$and $D^{-}$vanishing at infinity whose nontangential limits $\Phi^{ \pm}$from $D^{ \pm}$ are in $L_{n}^{p}(C)$ and satisfy the classical Riemann-Hilbert barrier problem $G \Phi^{+}=$ $\Phi^{-}+g$.

The problem under consideration in this paper is the question of when the operator $R_{G}$ is a Fredholm operator. Recall, a bounded linear operator on a Banach space is called Fredholm in case it has closed range and the null spaces of the operator and its adjoint are finite dimensional (Russian authors call such operators Noetherian). We will deal exclusively with the operators $R_{G}$ on $L_{n}^{2}(C)$ and will be preoccupied with local conditions on the symbol $G$ which determine whether or not $R_{G}$ is Fredholm.

There is a string of papers by I. B. Simonenko [13], see also the 6 references in [13], which establish that the question of deciding when $R_{G}$ is Fredholm is a local question, i.e. depends only on the behavior of the symbol $G$ in a neighborhood of each point on $C$. More recently, other authors Douglas and Widom [7], Douglas and Sarason [6] and Douglas ([4], [5]) have investigated similar local questions for the special case where the operator $R_{G}$ acts on $L^{2}(T)$; here, $T$ denotes the unit circle in $C$. These authors, notably in [6], [5], use function algebra and $C^{*}$-algebra methods.

Our concern will be with sybmols $G$ where the cluster behavior of $G$ at points $t_{0}$ in $C$ is particularly nice. We will assume that for some points $t_{0}$ in $C$ there are at most two matrices $X$ that satisfy

For every $\epsilon>0$ and neighborhood $N$ of $t_{0}$ the set $\{t \in C:\|G(t)-X\|<\epsilon\} \cap N$ has positive measure.

In equation (0.3) and in the remainder of this paper the norm of an $n \times n$ matrix $A$, denoted by $\|A\|$, will refer to the norm of the matrix considered as an operator on $\mathbf{C}^{n}$ with the Euclidean metric. 
In spite of the fact that the class of symbols under consideration appear restrictive our investigations have applications to systems of singular integral operators on $L^{2}(E)$, for measurable $E \subset C$, when the coefficients admit continuous extension to $C$. We briefly discuss this application in $\S 4$.

$\S 1$ of this paper is concerned with developing technical machinery aimed at reducing the problems to the generic case where $C=T$. $\$ 2$ gives sufficient conditions for the operators $R_{G}$ to be Fredholm. In $\S 3$ sufficient conditions are given which guarantee that the operator $R_{G}$ is not Fredholm. $\$ 4$ contains applications and remarks.

The author would like to thank Edward Azoff for valuable discussions, in particular, for simplification in the proof of Lemma 2.1.

1. Technical preliminaries. It is easy to verify that the operator $R_{G}$ : $L_{n}^{p}(C) \rightarrow L_{n}^{p}(C)$ is Fredholm if and only if the onerators $R_{G}: L_{n}^{p}\left(C_{i}\right) \rightarrow$ $L_{n}^{p}\left(C_{i}\right), i=0,1, \cdots, m$, are Fredholm; here, we have set $G_{i}$ equal to the restriction of $G$ to $C_{i}$ (see, e.g. Simonenko [13, Lemma 7]).

For $i=0,1, \cdots, m$, there is a conformal map $\beta_{i}$ from the bounded domain $D_{i}$ interior to $C_{i}$ to the unit disc in the complex plane. The maps $\beta_{i}$ extend to be one-to-one analytic maps of $C_{i}$ to $T$. (See Nehari [11, pp. 179 and 186].) The argument in Simonenko [13, Lemma 8] can be used to establish that $R_{G i}: L_{n}^{p}\left(C_{i}\right) \rightarrow L_{n}^{p}\left(C_{i}\right)$ is Fredholm if and only if $R_{G i \circ \beta \bar{i}^{1}}: L_{n}^{p}(\mathrm{~T}) \rightarrow$ $L_{n}^{p}(\mathrm{~T})$ is Fredholm.

The notations $H^{p} \quad(1 \leqslant p \leqslant \infty)$ will be used for the usual Hardy spaces on the unit circle and $H_{n}^{p}$ the $\mathbf{C}^{n}$-valued analogues. The map $f \rightarrow f^{+}$on $L_{n}^{2}(\mathrm{~T})$ is precisely the orthogonal projection of $L_{n}^{2}(\mathrm{~T})$ onto $H_{n}^{2}$. If $G$ is in $L_{M_{n}}^{\infty}(\mathrm{T})$, then the Toeplitz operator with symbol $G$ is the operator $T_{G}$ defined on $H_{n}^{2}$ by $T_{G} f=(G f)^{+}$. It is easy to establish that $T_{G}$ is Fredholm on $H_{n}^{2}$ if and only if $R_{G}$ is Fredholm on $L_{n}^{2}(\mathrm{~T})$.

Simonenko [13, Theorem 1] has established that the Riemann-Hilbert operator $R_{G}: L_{n}^{p}(C) \rightarrow L_{n}^{p}(C)$ is Fredholm if and only if for every $t_{0}$ in $C$ there is a neighborhood $N\left(t_{0}\right)$ and $G_{t_{0}}$ in $L_{M_{n}}^{\infty}(C)$ such that $R_{G_{t 0}}$ is Fredholm and $G \mid N\left(t_{0}\right)=G_{t_{0}}$. Douglas [5, Corollary 4.7] has given a $C^{*}$ algebra proof of the corresponding result on $H_{n}^{2}$. In view of the remarks in the first part of this section these localization results for Riemann-Hilbert operators on $L_{n}^{2}(C)$ and Toeplitz operators on $H_{n}^{2}$ are equivalent.

The following lemma appears in the scalar case in Brown and Halmos [1]. The simple proof, which we include for completeness, is due to Widom.

Lemma 1.1. Suppose $G$ is in $L_{M_{n}}^{\infty}(\mathrm{T})$ and for some $\epsilon>0, \operatorname{Re} G(t) \geqslant$ eI a.e. Then $T_{G}$ is invertible. 
Proof. For $\delta>0$ small, we have almost everywhere

$$
\|I-\delta G(t)\| \leqslant\|I-\delta \operatorname{Re} G(t)\|+\delta\|G(t)\|<1 .
$$

It follows that $T_{\delta G}=\delta T_{G}$ is invertible. This completes the proof.

2. $R_{G}$ Fredholm. Throughout this section it will be assumed that at every point $t_{0} \in C$ the symbol $G$ has at most two cluster values $G_{1}\left(t_{0}\right), G_{2}\left(t_{0}\right)$ satisfying (0.3).

LEMma 2.1. Suppose that for some $t_{0}$ in $C$

$$
\operatorname{det}\left[\lambda G_{1}\left(t_{0}\right)+(1-\lambda) G_{2}\left(t_{0}\right)\right] \neq 0, \text { for } 0 \leqslant \lambda \leqslant 1 \text {. }
$$

Then there is a neighborhood $N_{t_{0}}$ of $t_{0}, \epsilon>0$, and invertible matrices $P, Q$ such that for almost every $t$ in $N_{t_{0}}, \operatorname{Re}(P G(t) Q) \geqslant \epsilon I$.

Proof. Set $M=G_{1}\left(t_{0}\right) G_{2}^{-1}\left(t_{0}\right)$. Obviously, $\operatorname{det}[\lambda+(1-\lambda) M] \neq 0 \quad(0 \leqslant$ $\lambda \leqslant 1)$. There exists an invertible matrix $W$ such that $W M W^{-1}=S+N$ is in Jordan canonical form, where $S$ is diagonal and $N$ is a nilpotent. Since $\operatorname{det}[\lambda I+(1-\lambda) S] \neq 0(0 \leqslant \lambda \leqslant 1)$ it is clear that there is a unitary matrix $U$ (in fact, diagonal) such that $\operatorname{Re} U \geqslant \alpha I$ and $\operatorname{Re} U S \geqslant \alpha I$, for some $\alpha>0$. The nilpotent $N$ is similar to $\beta N$, for any $\beta \neq 0$. This follows because $N$ and $\beta N$ have the same Jordan form. Indeed, by considering the block form of $S$ and $N$, for any $\beta>0$ there exists an invertible matrix $V_{\beta}$ such that $V_{\beta} N V_{\beta}^{-1}=\beta N$ and $V_{\beta} S V_{\beta}^{-1}=S$. Clearly, by taking $\beta$ small enough, we have $\operatorname{Re} U \geqslant \gamma I$ and $\operatorname{Re}\left(U V_{\beta} W M W^{-1} V_{\beta}^{-1}\right) \geqslant \gamma I$, for some $\gamma>0$. In other words, if $P=U V_{\beta} W$ and $Q=G_{1}^{-1}\left(t_{0}\right) W^{-1} V_{\beta}^{-1}$, then $\operatorname{Re}\left(P G_{i}\left(t_{0}\right) Q\right) \geqslant \gamma I$, for $i=$ $1,2$.

The lemma follows since almost every value of $G(t)$ in a neighborhood of $t_{0}$ can be made arbitrarily close to $G_{1}\left(t_{0}\right)$ or $G_{2}\left(t_{0}\right)$ by taking the neighborhood small enough. This completes the proof.

Douglas and Widom [7] have asked the following question: Given a compact convex set $K$ of invertible matrices do there exist invertible matrices $P$, $Q$ such that $\operatorname{Re} P K Q \geqslant \epsilon I>0$, for all $K$ in $K$ ? Lemma 2.1 answers this question affirmatively when $K$ is a line segment.

The main result of this section is

THEOREM 2.1. Let $G$ be in $L_{M_{n}}^{\infty}(C)$ and suppose that for every $t_{0}$ in $C$ there are at most two cluster values $G_{1}\left(t_{0}\right), G_{2}\left(t_{0}\right)$ satisfying $(0.3)$. Assume further, that $\operatorname{det}\left[\lambda G_{1}\left(t_{0}\right)+(1-\lambda) G_{2}\left(t_{0}\right)\right] \neq 0$, for all $t_{0}$ in $C$ and $0 \leqslant \lambda \leqslant 1$. Then the operator $R_{G}$ defined by $(0.2)$ on $L_{n}^{2}(C)$ is Fredholm.

Proof. In view of the remarks in $\S 1$ we can assume $C=T$ and establish that $T_{G}$ is Fredholm. It follows from Lemma 2.1 that for every point $t_{0}$ in 
T there exist invertible matrices $P_{t_{0}}$ and $Q_{t_{0}}$ and a neighborhood $N_{t_{0}}$ such that $H(t)=P_{t_{0}} G(t) Q_{t_{0}}$ satisfies $\operatorname{Re} H(t) \geqslant \epsilon I>0$, for almost all $t$ in $N_{t_{0}}$. Define,

$$
G_{t_{0}}(t)=\left\{\begin{array}{l}
G(t), \quad t \in N_{t_{0}} \\
G_{1}\left(t_{0}\right), \quad t \notin N_{t_{0}}
\end{array}\right.
$$

and

$$
H_{t_{0}}(t)= \begin{cases}H(t), & t \in N_{t_{0}} \\ P_{t_{0}} G_{1}\left(t_{0}\right) Q_{t_{0}}, & t \notin N_{t_{0}}\end{cases}
$$

Then

$$
T_{G_{t_{0}}}=T_{P_{t_{0}}} T_{H_{t_{0}}} T_{Q_{t_{0}}}
$$

and since $T_{P_{t_{0}}}, T_{Q_{t_{0}}}$ are obviously invertible and $T_{H_{t_{0}}}$ is invertible by Lemma 1.1, it follows that $T_{G_{0}}$ is invertible. Consequently, $T_{G}$ is locally Fredholm and, therefore, by Simonenko [13, Theorem 1] or Dougtas [5, Corollary 4.7] the operator $T_{G}$ is Fredholm. This completes the proof.

3. $R_{G}$ not-Fredholm. In this section we will obtain sufficient conditions on $G$ which guarantee that the operator $R_{G}$ on $L_{n}^{2}(C)$ is not-Fredholm. The investigation involves a localization to Hardy spaces of representing measures for $H^{\infty}$. This technique was first used by D. Sarason (private communication) in the study of Toeplitz operators on $H^{2}$.

The maximal ideal space of the algebra $L^{\infty}(\mathrm{T})$ is denoted by $X$. The space $X$ is normally fibered over the unit circle, so that, $X=\bigcup_{|\lambda|=1} X_{\lambda}$; where, the fibre $X_{\lambda}$ consists of the homomorphisms of $L^{\infty}(\mathbf{T})$ that assign the value $\lambda$ to the function $\chi\left(e^{i t}\right)=e^{i t}$. The notation $Y$ will be used for the maximal ideal space of $H^{\infty}$. For $\lambda$ in $\mathrm{T}$, the fibre $Y_{\lambda}$ consists of the homomorphisms of $H^{\infty}$ which assign the value $\lambda$ to $\chi$. The "algebra on the fibre" $A_{\lambda}$ is the restriction of $\hat{H}^{\infty}$ (the space of Gelfand transforms) to the fibre $X_{\lambda}$.

We will be using many of the properties of $X, Y, X_{\lambda}, Y_{\lambda}$ and $A_{\lambda}$. Hoffman's book [9, Chapter 10] is the best reference for these properties. Indeed, we will need the following facts:

(i) $X$ is the Shilov boundary of the algebra $H^{\infty}$ and if $\gamma$ is a homomorphism of $H^{\infty}$ in the fibre $Y_{\lambda}$, then $\gamma$ has a unique representing measure supported on $X_{\lambda}$.

(ii) $A_{\lambda}$ is a closed subalgebra of $C\left(X_{\lambda}\right)$ whose maximal ideal space is $Y_{\lambda}$ and whose Shilov boundary is $X_{\lambda}$. 
(iii) $Y_{\lambda}$ is connected.

(iv) If $W, V$ are disjoint closed sets in $X_{\lambda}$, then there is an $F$ in $A_{\lambda}$ such that $\left.F\right|_{W}=0$ and $|F|=1$ on $V$.

Hardy spaces of representing measures have been studied by many authors (see, Gamelin [8, Chapter 4] and the references there). Toeplitz operators on Hardy spaces of representing measures were studied by Devinatz [3]. For our purposes we will need the $\mathbf{C}^{n}$-valued Hardy spaces of a representing measure $\mu$ of $H^{\infty}$ supported on a fibre $X_{\lambda}$. For convenience we assume the measure $\mu$ is supported on the fibre $X_{1}$. Let $L_{n}^{p}(\mu)(1 \leqslant p \leqslant \infty)$ denote the $\mathbf{C}^{n}$. valued Lebesgue spaces. The notation $H_{n}^{p}(\mu)(1 \leqslant p<\infty)$ will be used for the closure of $A_{1} \otimes \mathbf{C}^{n}$ in $L_{n}^{p}(\mu)$ and $H_{n}^{\infty}(\mu)$ the weak*-closure of $A_{1} \otimes \mathbf{C}^{n}$ in $L_{n}^{\infty}(\mu)$. If $P$ denotes the orthogonal projection of $L_{n}^{2}(\mu)$ onto $H_{n}^{2}(\mu)$ and $\Phi \in L_{M_{n}}^{\infty}(\mu)$, then the Toeplitz operator $T_{\Phi}$ on $H_{n}^{2}(\mu)$ is defined by $T_{\Phi} f=$ $P \Phi f$.

Assume now that the fibre $X_{1}$ is the disjoint union of a fixed pair of nonempty closed sets $X_{1}^{+}$and $X_{1}^{-}$. It follows easily from properties (i) and (iii) above that there is a homomorphism of $H^{\infty}$ with measure $\mu_{0}$ supported on $X_{1}$ such that $\mu_{0}\left(X_{1}^{ \pm}\right)>0$.

Let $\mathbb{Q}$ be the subalgebra of $L^{\infty}\left(\mu_{0}\right)$ consisting of functions which are constant a.e. $\left[\mu_{0}\right]$ on $X_{1}^{ \pm}$. We will be concerned with the collection $B_{M_{n}}$ consisting of symbols $G$ in $L_{M_{n}}^{\infty}\left(\mu_{0}\right)$ which can be factored in the form $G=G_{1} G_{2} G_{3}$, where $G_{1}^{*}, G_{3}$ are invertible elements in $\left(A_{1}\right)_{M_{n}}$ and $G_{2}$ is in $\mathbb{C}_{M_{n}}$.

Before stating a theorem on Toeplitz operators with symbols in $B_{M_{n}}$ we prove

Lemma 3.1. Let $\Phi$ be in $\mathfrak{C}_{M_{n}}$. If $\operatorname{det} \Phi=0$ on $X_{1}^{+}$or $X_{1}^{-}$, then $T_{\Phi}$ is not left-invertible on $H_{n}^{2}\left(\mu_{0}\right)$.

Proof. Assume without loss of generality that $\operatorname{det} \Phi=0$ on $X_{1}^{+}$. It is easy to see that there is an invertible matrix $S$ such that the first column of $\Psi=S \Phi S^{-1}$ is zero on $X_{1}^{+}$. Since, $T_{\Psi}=T_{S} T_{\Phi} T_{S^{-1}}$ and $T_{S}, T_{S^{-1}}$ are invertible it suffices to show that $T_{\Psi}$ is not left-invertible. By property (iv) there exists an $f$ in $H_{n}^{2}\left(\mu_{0}\right), f \neq 0$ and $f\left(X_{1}^{-}\right)=0$. Let $F$ be the $\mathbf{C}^{n}$-valued $H^{2}\left(\mu_{0}\right)$ function whose first entry is $f$ and remaining entries are zero.

Clearly, $T_{\Psi} F=0$ and this completes the proof.

The following theorem is essentially a result on vector valued "self-adjoint" Toeplitz operators on $H_{n}^{2}\left(\mu_{0}\right)$.

THEOREM 3.1. Let $G$ be in $B_{M_{n}}, G=G_{1} G_{2} G_{3}$, where $G_{1}^{*}, G_{3}$ are invertible in $\left(A_{1}\right)_{M_{n}}$ and $G_{2}$ is constant with values $G_{2}^{ \pm}$on $X_{1}^{ \pm}$. If 
$\operatorname{det}\left[\lambda_{0} G_{2}^{+}+\left(1-\lambda_{0}\right) G_{2}^{-}\right]=0$, for some $\lambda_{0}, 0 \leqslant \lambda_{0} \leqslant 1$, then the operator $T_{G}$ on $H_{n}^{2}\left(\mu_{0}\right)$ is not left-invertible.

Proof. Obviously, it suffices to show $T_{G_{2}}$ is not left-invertible. We can assume by the preceding lemma that $G_{2}^{ \pm}$are invertible. Set $M=G_{2}\left(G_{2}^{+}\right)^{-1}$. Clearly, $M$ is in $\mathbb{Q}_{M_{n}}$ and $T_{G_{2}}$ is left-invertible if and only if $T_{M}$ is left invertible. On $X_{1}^{-}$the symbol $M$ equals $M^{-}=G_{2}^{-}\left(G_{2}^{+}\right)^{-1}$. Choose an invertible $S$ so that $S M S^{-1}$ is in the Jordan canonical form. It follows that $N=$ $S M S^{-1}$ is in $\mathfrak{Q}_{M_{n}}$ and is in Jordan canonical form at each point of $X_{1}$. Moreover, $T_{G_{2}}$ is left-invertible if and only if $T_{N}$ is left-invertible. If $N^{ \pm}$denote the values of $N$ on $X_{1}^{ \pm}\left(N^{+}=\right.$identity) and if the diagonal entries of $N^{-}$are $\eta_{11}^{-}, \eta_{22}^{-}, \cdots, \eta_{n n}^{-}$, then by rechoosing $S$ if necessary we can assume $\lambda_{0}+$ $\left(1-\lambda_{0}\right) \eta_{11}^{-}=0$. Consider the self-adjoint Toeplitz operator $T_{\varphi}$ on $H^{2}\left(\mu_{0}\right)$ where $\varphi=1$ on $X_{1}^{+}$and $\varphi=\eta_{11}^{-}$on $X_{2}^{-}$. The operator $T_{\varphi}$ is not leftinvertible. (Devinatz, [3., Theorem 1]. Actually, Devinatz's theorem is only proven for the case of representing measures of Dirichlet algebra, however, the proof lifts to the case under consideration here.) The triangular form of $N$ makes it obvious that $T_{N}$ is not left-invertible. This completes the proof.

The next step is to obtain a theorem for the operators $R_{G}$ on $L_{n}^{2}(C)$ from Theorem 3.1. We work first with the case where $C=\mathbf{T}$.

Again it is assumed that the fibre $X_{1}$ has been partitioned into a fixed pair of nonempty closed sets $X_{1}^{ \pm}$and $\mu_{0}$ is a representing measure of a homomorphism of $H^{\infty}$ supported in $X_{1}$ such that $\mu_{0}\left(X_{1}^{ \pm}\right)>0$. The notation $\widetilde{\mathscr{C}}$ will denote the subalgebra of $L^{\infty}(\mathrm{T})$ consisting of functions whose Gelfand transforms are constant on $X_{1}^{ \pm}$. Clearly, $(\widetilde{\mathscr{Q}})^{\wedge} \subset \mathbb{Q}$. If $G$ is in $\widetilde{\mathscr{U}}_{M_{n}}$, then $G^{ \pm}$will denote the values of $\hat{G} \mid X_{1}^{ \pm}$. The main theorem concerning Toeplitz operators with symbols in $\widetilde{\mathbb{Q}}_{M_{n}}$ is the following:

Theorem 3.2. Assume that $G$ is in $\widetilde{\mathbb{Q}}_{M_{n}}$ and that for some $\lambda_{0}, 0 \leqslant \lambda_{0} \leqslant 1$, $\operatorname{det}\left[\lambda_{0} G^{+}+\left(1-\lambda_{0}\right) G^{-}\right]=0$. Then $T_{G}$ is not-Fredholm on $H_{n}^{2}$.

The proof will involve some results of Rabindranathan [12] which we now state.

LEMMA 3.2 (Rabindranathan [12, Lemma 4.1]). Let $\Phi$ be an invertible element in $L_{M_{n}}^{\infty}(\mathrm{T})$. Then $\Phi=U A$, where $A$ is an invertible element in $H_{M_{n}}^{\infty}$ and $U$ is unitary valued a.e.

The following result is a matrix analogue of a result of Douglas and Sarason [6]. It can easily be derived from Lemma 4.3 of Rabindranathan [12].

LEMma 3.3. Let $U$ be a unitary valued element of $L_{M_{n}}^{\infty}(\mathrm{T})$. Then $T_{U}$ on $H_{n}^{2}(\mathrm{~T})$ is left-Fredholm if and only if there is an $F$ in $\left(H^{\infty}+C\right)_{M_{n}}$ (here, 
$C$ denotes the continuous functions on $\mathrm{T})$ such that

$$
\begin{gathered}
\|U-F\|_{\infty} \equiv \operatorname{ess} \sup \left\|U\left(e^{i t}\right)-F\left(e^{i t}\right)\right\|<1 . \\
e^{i t} \in \mathbf{T}
\end{gathered}
$$

The final result needed to prove Theorem 3.2 is the following elementary lemma.

LEMMA 3.4. Suppose $\mu$ is a representing measure of a homomorphism of $H^{\infty}$ supported on the fibre $X_{1}$. Let $U$ be in $L_{M_{n}}^{\infty}(\mu)$ and unitary valued. If there exists an $F$ in $H_{M_{n}}^{\infty}(\mu)$ such that

then $T_{U}$ is left-invertible.

$$
\|U-F\|_{\infty} \equiv \underset{\gamma \in X_{1}}{\operatorname{ess} \sup \|U(\gamma)-F(\gamma)\|<1,}
$$

Proof of Theorem 3.2. Suppose the operator $T_{G}$ is Fredholm. Then $G$ is an invertible element in $L_{M_{n}}^{\infty}(\mathrm{T})$ and, therefore, by Lemma 3.2, $G=U A$ where $U$ is unitary and $A$ is an invertible element in $H_{M_{n}}^{\infty}$. The operator $T_{U}$ is certainly left-Fredholm and, hence, there exists by Lemma 3.3, an $F$ in $\left(H^{\infty}+C\right)_{M_{n}}$ such that $|U-F|_{\infty}<1$. From the equality

$$
\|U-F\|_{\infty}=\sup _{\gamma \in X}\|\hat{U}(\gamma)-\hat{F}(\gamma)\|
$$

and the fact that $\left.\hat{C}\right|_{X_{1}} \subset A_{1}$, we obtain an $H$ in $\left(A_{1}\right)_{M_{n}}$ such that

$$
\sup _{\gamma \in X_{1}}\|\hat{U}(\gamma)-H(\gamma)\|<1
$$

This says that the operator $T_{\hat{U}}$ is left-invertible on $H_{n}^{2}(\mu)$ for any representing measure $\mu$ for a homomorphism of $H^{\infty}$ supported on $X_{1}$. In particular, $T \hat{U}$ is left-invertible on $H_{n}^{2}\left(\mu_{0}\right)$. However, $\hat{U}=\hat{G} \hat{A}^{-1}$, where $\hat{A}^{-1}$ is invertible in $\left(A_{1}\right)_{M_{n}}$ and $\hat{G}$ is in $\mathbb{Q}_{M_{n}}$. (In other words $\hat{U}$ is in $B_{M_{n}}$.) From the hypothesis $\operatorname{det}\left[\lambda_{0} G^{+}+\left(1-\lambda_{0}\right) G^{-}\right]=0$ and Theorem 3.1 we conclude that $T_{\hat{G}}$ is not left-invertible on $H_{n}^{2}\left(\mu_{0}\right)$. This contradiction completes the proof.

In case $C$ is the union of the contours $C_{0}, C_{1}, \cdots, C_{m}$, then the map $\beta: C \rightarrow \mathrm{T}\left(\beta \mid C_{i} \equiv \beta_{i}\right)$ induces a natural "fibration" of $L^{\infty}(C)$ at points of $C$. Indeed, if $t_{0}$ is in $C$, every partition of $X_{\beta\left(t_{0}\right)}$ into a pair of closed sets $X_{\beta\left(t_{0}\right)}^{ \pm}$and corresponding algebra $\widetilde{\mathscr{C}}^{\beta}\left(t_{0}\right) \subset L^{\infty}(\mathrm{T})$ consisting of functions whose Gelfand transforms are constant on $X_{\beta\left(t_{0}\right)}^{ \pm}$, gives rise to the algebra $\widetilde{\mathbb{Q}}^{t_{0}}$ consisting of functions $f$ in $L^{\infty}(C)$ such that $f \circ \beta^{-1}$ is in $\widetilde{\mathbb{Q}}^{\beta\left(t_{0}\right)}$. The following corollary is immediate:

Corollary 3.1. Let $t_{0}$ be in $C$. Assume $G$ is in $\widetilde{\mathbb{Q}}_{M_{n}}^{t_{0}}$ (for some decomposition of $\left.X_{\beta\left(t_{0}\right)}\right)$. Let $G^{ \pm}$be the values of $G \circ \beta^{-1}$ on $X_{\beta\left(t_{0}\right)}^{ \pm}$. If 
$\operatorname{det}\left[\lambda_{0} G^{+}+\left(1-\lambda_{0}\right) G^{-}\right]=0$, for some $\lambda_{0}, 0 \leqslant \lambda_{0} \leqslant 1$, then $R_{G}$ is notFredholm on $L_{n}^{2}(C)$.

4. Systems of singular integral equations. Let $E$ be a bounded measurable subset of $C$ and let $A, B$ be in $L_{M_{n}}^{\infty}(E)$. It will always be assumed that $A \pm B$ are invertible in $L_{M_{n}}^{\infty}(E)$. Consider the singular integral operator $S$ on $L_{n}^{2}(E)$ defined by

$$
S f(s)=A(s) f(s)+B(s) Q f(s), \quad s \in E .
$$

It follows from the Plemelj identities $(0.1)$ that

$$
S f=(A+B) f^{+}+(A-B) f^{-}, \quad f \text { in } L_{n}^{2}(E) .
$$

Consider the symbol $G$ in $L_{M_{n}}^{\infty}(C)$ defined by

$$
G(s)= \begin{cases}(B-A)^{-1}(A+B)(s), & s \in E, \\ I, & s \notin E .\end{cases}
$$

The following lemma is easily established.

LEMMA 4.1. Let $S$ denote the singular integral operator defined by (4.1) on $L_{n}^{2}(E)$ and $G$ the symbol in $L_{M_{n}}^{\infty}(C)$ defined in (4.2). Then $S$ is Fredholm on $L_{n}^{2}(E)$ if and only if $R_{G}$ is Fredholm on $L_{n}^{2}(C)$.

The technique of associating the barrier operator $R_{G}$, where the symbol $G$. is defined by (3.2), with the operator $S$ on $L_{n}^{2}(E)$ was exploited for the case $n=1$ in [2]. In fact, the results in [2] are motivation for this paper.

Suppose the matrix symbols $A, B$ appearing in $L_{M_{n}}^{\infty}(E)$ are restrictions of continuous matrix functions $\hat{A}, \hat{B}$ on $C$ to $E$. In this case the cluster behavior of the symbol $G$ appearing in (4.2) is easy to describe. Let $t_{0}$ be in $C$. The matrix $\hat{G}\left(t_{0}\right)=(\hat{B}-\hat{A})^{-1}(\hat{A}+\hat{B})\left(t_{0}\right)$ is a cluster value of $G$ at $t_{0}$ if and only if every neighborhood of $t_{0}$ intersects $E$ in a set of positive measure. The identity matrix is a cluster value of $G$ at $t_{0}$ if and only if every neighborhood of $t_{0}$ intersects the complement of $E$ in a set of positive measure. The matrices $\hat{G}\left(t_{0}\right)$ and $I$ are the only possible cluster values. Further, if $\beta: C \rightarrow T$ is the map introduced in $\S 1$ and $\chi_{\beta_{i}(E)}$ denotes the characteristic function of $\beta_{i}(E)$, then, for $t_{0}$ in $C_{i}, X_{\beta_{i}\left(t_{0}\right)}$ has a natural partition into the pair of disjoint closed sets

$$
X_{\beta_{i}\left(t_{0}\right)}^{+}=\left\{\gamma \in X_{\beta_{i}\left(t_{0}\right)}: \hat{\chi}_{\beta_{i}(E)}=1\right\}
$$

and

$$
X_{\beta_{i}\left(t_{0}\right)}^{-}=\left\{\gamma \in X_{\beta_{i}\left(t_{0}\right)}: \hat{\chi}_{\beta_{i}(E)}=0\right\}
$$

The symbol $G$ belongs to the algebra $\widetilde{\mathbb{C}}_{M_{n}}^{t_{0}}$ relative to this decomposition. 
Our final result is a direct consequence of Theorem 2.1 and Corollary 3.1.

THEOREM 4.1. Let $S$ be the singular integral operator defined in (4.1) on $L_{n}^{2}(E)$. Assume that $A, B$ are the restrictions of continuous matrix functions $\hat{A}$, $\hat{B}$ defined on $C$ to $E$. The operator $S$ is Fredholm if and only if for every point $t_{0}$ in $C$ such that every neighborhood of $t_{0}$ intersects both $E$ and its complement in a set of positive measure

$$
\operatorname{det}\left[\lambda \hat{G}\left(t_{0}\right)+(1-\lambda) \eta\right] \neq 0, \quad 0 \leqslant \lambda \leqslant 1 .
$$

REMARKS. Nothing has been said about the index of the operator $R_{G}$. Except in the routine case where the symbol $G$ is upper triangular we have been unable to give a natural computation of the index comparable to the result in [7]. One obstacle is the impossibility of putting a continuous symbol into upper triangular form in a continuous manner.

Even in the scalar case, when the symbol $G$ is constant on each element of a partition of $X_{1}$ into more than two disjoint closed sets, no result corre, sponding to Corollary 3.1 is known.

\section{REFERENCES}

1. A. Brown and P. R. Halmos, Algebraic properties of Toeplitz operators, J. Reine Angew. Math. 213 (1963/64), 89-102. MR 28 \#3350; 30, 1205.

2. A. Devinatz, Toeplitz operators on $H^{2}$ spaces, Trans. Amer. Math. Soc. 112 (1964), 304-317. MR 29 \#477.

3. K. Clancey, The essential spectrum of a class of singular integral operators, Amer. J. Math. (to appear).

4. R. G. Douglas, Banach algebra techniques in operator theory, Academic Press, New York, 1972.

5. - Banach algebra techniques in the theory of Toeplitz operators, Conference Board of the Math. Sci., Regional Conference Ser. in Math., no. 15, Amer. Math. Soc., Providence, R.I., 1973.

6. R. G. Douglas and D. E. Sarason, Fredholm Toeplitz operators, Proc. Amer. Math. Soc. 26 (1970), 117-120. MR 41 \#4275.

7. R. G. Douglas and H. Widom, Toeplitz operators with locally sectorial symbols, Indiana Univ. Math. J. 20 (1970/71), 385-388. MR 41 \#9024.

8. T. W. Gamelin, Uniform algebras, Prentice-Hall Series in Modern Analysis, PrenticeHall, Englewood Cliffs, N. J., 1969.

9. K. Hoffman, Banach spaces of analytic functions, Prentice-Hall Series in Modern Analysis, Prentice-Hall, Englewood Cliffs, N. J., 1962. MR 24 \#A2844.

10. N. I. Mushelisvili, Singular integral equations. Boundary problems of function theory and their application to mathematical physics, OGIZ, Moscow, 1946; English transl., Noordhoff, Groningen, 1953. MR 8, 586; 15, 434.

11. Z. Nehari, Conformal mapping, McGraw-Hill, New York, 1952. MR 13, 640. 
12. M. Rabindranathan, On the inversion of Toeplitz operators, J. Math. Mech. 19 (1969/70), 195-206. MR 40 \#4785.

13. I. B. Simonenko, Some general questions on the theory of the Riemann boundary problem, Izv. Akad. Nauk SSSR Ser. Mat. 2 (1968), 1138-1146 = Math. USSR Izv. 2 (1968), 1091-1099. MR 38 \#3447.

14. A. Zygmund, Trigonometric series. Vol. 1, 2nd rev. ed., Cambridge Univ. Press, New York, 1959. MR 21 \#6498.

DEPARTMENT OF MATHEMATICS, UNIVERSITY OF GEORGIA, ATHENS, GEORGIA 30602 